Post-print of: AIDS, 14 January 2012 - Volume 26 - Issue 2 - p 127-137

doi: 10.1097/QAD.0b013e32834e1567

\title{
A glycomimetic compound inhibits DC-SIGN-mediated HIV infection in cellular and cervical explant models
}

Berzi, Angela (a); Reina, José J. (d); Ottria, Roberta (d); Sutkeviciute, leva (e,f,g); Antonazzo, Patrizio (i); Sanchez-Navarro, Macarena (j); Chabrol, Eric (e,f,g); Biasin, Mara (a); Trabattoni, Daria (a); Cetin, Irene (i); Rojo, Javier (j); Fieschi, Franck (e,f,g,h); Bernardi, Anna (d,k); Clerici, Mario (b,c)

(a) Chair of Immunology, Department of Clinical Sciences L. Sacco

(b) Chair of Immunology, Department of Biomedical Sciences and Technologies, University of Milan, Segrate

(c)Don C. Gnocchi Foundation IRCSS

(d) Department of Organic and Industrial Chemistry, University of Milan, Milan Italy

(e) Institut de Biologie Structurale, Université Joseph Fourier

(f) CEA, DSV

(g) CNRS, UMR 5075, Grenoble

(h) Institut Universitaire de France, Paris, France

(i) Unit of Obstetrics and Gynaecology, Department of Clinical Sciences L. Sacco, University of Milan, Milan, Italy

\begin{abstract}
Objective: Dendritic cell-specific intercellular adhesion molecule (ICAM)-3 grabbing nonintegrin (DC-SIGN) participates in the initial stages of sexually transmitted HIV-1 infection by recognizing highly mannosylated structures presented in multiple copies on HIV-1 gp120 and promoting virus dissemination. Inhibition of HIV interaction with DC-SIGN thus represents a potential therapeutic approach for viral entry inhibition at the mucosal level.

Design: Herein we evaluate the efficacy in inhibiting HIV-1 infection and the potential toxicity of a multimeric glycomimetic DC-SIGN ligand (Dendron 12).
\end{abstract}


Methods: The ability of Dendron 12 to block HIV-1 infection was assessed in cellular and human cervical explant models. Selectivity of Dendron 12 towards DC-SIGN and langerin was evaluated by surface plasmon resonance studies. $\beta$ chemokine production following stimulation with Dendron 12 was also analyzed. Toxicity of the compound was evaluated in cellular and tissue models.

Results: Dendron 12 averted HIV-1 trans infection of CD4+ T lymphocytes in presence of elevated viral loads and prevented HIV-1 infection of human cervical tissues, under conditions mimicking compromised epithelial integrity, by multiple clades of R5 and X4 tropic viruses. Treatment with Dendron 12 did not interfere with the activity of langerin and also significantly elicited the production of the $\beta$ chemokines MIP- $1 \alpha$, MIP-1 $\beta$ and RANTES.

Conclusion: Dendron 12 thus inhibits HIV-1 infection by competition with binding of HIV to DCSIGN and stimulation of $\beta$-chemokine production. Dendron 12 represents a promising lead compound for the development of anti-HIV topical microbicides.

\section{Introduction}

HIV remains one of the leading causes of mortality and morbidity [1]. As the vast majority of HIV-1 infections occur via sexual transmission through mucosal surfaces, the development of vaginal and rectal topical microbicides represents a promising approach to prevent sexually transmitted HIV-1 infection.

Dendritic cell-specific intercellular adhesion molecule-3 grabbing nonintegrin (DC-SIGN) is involved in the initial step of HIV-1 sexually transmitted infection and it may be considered a promising therapeutic target $[2,3]$.

Myeloid immature dendritic cells located in mucosal tissue of vagina, cervix and rectum express DC-SIGN and are among the first cells to encounter sexually transmitted HIV [4-6]. Upon HIV-1 binding to DC-SIGN, the virus escapes (at least partially) degradation into lysosomes. Rather, it is internalized into endosomes and multivescicolar bodies, in which it is protected from degradation and retained in a high infective state [7-9]. Dendritic cells transmit the virus in trans to adjacent CD4+ $\mathrm{T}$ lymphocytes in genital mucosae or, after migration to lymphoid tissue, to CD4+ $T$ lymphocytes resident in lymphoid tissue, promoting HIV-1 dissemination [10,11]. DC-SIGN, facilitating HIV-1 interaction with CD4 and co-receptors, also enhances dendritic cell infection in cis, that results in long-term transmission of HIV $[12,13]$.

In addition, binding of HIV-1 to DC-SIGN activates signaling pathways that modulate Toll-like receptor signaling, inducing immunosuppressive responses and triggering HIV replication and transmission [14-17].

DC-SIGN specifically recognizes the high mannose glycan (Man9), presented in multiple copies on HIV-1 gp120 [8,18]. Fragments of Man9 terminated by a di-mannoside or a tri-mannoside bind to DC-SIGN almost as efficiently as the entire Man9[19]. Chemically synthesized analogs of (Man)9 terminal di-saccharides and tri-saccharides are more resistant to hydrolysis by 
glycosydases than the corresponding natural oligosaccharides and interact efficiently with DCSIGN [20]. These analogs can be linked to tetravalent (dendrons) scaffolds to obtain compounds endowed with stronger binding affinities to DC-SIGN $[21,22]$. Such compounds can compete with binding of HIV gp120 to DC-SIGN and are suitable for the development of new anti-HIV microbicides.

We recently demonstrated that a tetravalent dendron containing four copies of a linear pseudo-mannotrioside (Dendron 12) was able to inhibit HIV-1 trans infection of CD4+ T lymphocytes [23].

Experimental models based on infection with HIV of explants taken from human uterine cervix, albeit with some limitations, allow a better approximation of the conditions in vivo compared to cellular models [24-27]. Thus, in this study a cervical explant model was exploited to assess the efficacy in inhibiting HIV-1 infection and the toxicity of Dendron 12, with the purpose of evaluating if the compound is a suitable candidate for the development as topical microbicide.

\section{Material and methods}

\section{Cell culture}

B-THP1 and B-THP1/DC-SIGN cells (contributed by Drs Li Wu and Vinet N. KevalRamani) were cultured as described [23].

\section{Virus}

The following HIV-1 strains were used: BaL (contributed by Drs. S. Gartner, M. Popovic and R. Gallo; DU174 (Source: Dr L Morris); the R5 and X4 tropic primary isolates $8 \mathrm{~g}$ and DPMVF (provided by Professor Stefano Aquaro).

Inhibition of HIV infection in trans

Human CD4+ T lymphocytes were purified from peripheral blood of volunteer healthy donors following written consent, and activated as described [23]. Dendron 12, synthesized as described [23], was diluted to desired concentration into culture medium [Roswell Park Memorial Institute (RPMI) 160 with 20\% fetal bovine serum (FBS), penicillin and streptomycin and L-glutamine, all from Euroclone, Siziano, Italy]. B-THP1/DC-SIGN or B-THP1 cells (106 cells $/ \mathrm{ml}$ ) were preincubated with Dendron $12(250 \mu \mathrm{mol} / \mathrm{l})$, or culture medium alone for 30 min prior to exposure to BaL (virus titer ranging from 5 TCID50 to $80 \mathrm{TCID50}$ ), in the continued presence of the inhibitor $3 \mathrm{~h}$ at $37^{\circ} \mathrm{C}$. After extensive washing, B-THP1/DC-SIGN cells were co-cultured with activated human $\mathrm{CD} 4+\mathrm{T}$ cells as previously described [23].

\section{Human cervical explants infection}

Cervical tissue was obtained, following written informed consent, from premenopausal women, HIV, hepatitis B virus (HBV) and hepatitis C virus (HCV) seronegative and without current genital infection, undergoing therapeutic hysterectomy at Unit of Obstetrics and Gynaecology of Sacco Hospital (Milan, Italy). The study was approved by the local ethic 
committee and conducted in compliance with international guidelines and local laws. Endocervical $3 \mathrm{~mm} \times 3 \mathrm{~mm}$ explant biopsies comprised both epithelium and stromal tissue.

Within $1 \mathrm{~h}$ after obtaining tissues the explants were extensively washed. Then explants were either immediately treated with the Dendron 12 and infected with R5 tropic strains or were prestimulated for 2 days in presence of IL-2 (R\&D systems, Minneapolis, Minnesota, USA) and phytohaemagglutinin (PHA) (Sigma-Aldrich, Saint Louis, Missouri, USA) prior to X4 tropic DPMVF exposure.

Explants were pretreated $30 \mathrm{~min}$ at $37^{\circ} \mathrm{C}$ with different concentrations of Dendron 12 . Afterwards explants were exposed to HIV-1 BaL, DU174 (both $2.6 \times 104$ TCID50), $8 \mathrm{~g}$ or DPMVF (both $104 \mathrm{TCID50}$ ) in the continued presence of the compound $3 \mathrm{~h}$ at $37^{\circ} \mathrm{C}$. Subsequently explants were washed with RPMI. Unstimulated explants were cultured in RPMI medium supplemented with $20 \% \mathrm{FBS}$, penicillin and streptomycin, L-glutamine (Euroclone) and gentamycin (Sigma-Aldrich) at $37^{\circ} \mathrm{C}$ and $5 \% \mathrm{CO}$. Stimulated explants were cultured under the same conditions in presence of IL-2. Supernatants were collected 3 and 7 days post infection.

\section{p24 ELISA}

p24 concentration in the supernatants was assayed by Alliance HIV-1 p24 Antigen kit (Perkin Elmer, Waltham, Massachusetts, USA). Plates were read using the IMark microplate reader equipped with Microplate Manager 6 software (Biorad, Segrate, Italy).

\section{Toxicity on peripheral blood mononuclear cells}

Peripheral blood mononuclear cells (PBMC), purified from peripheral blood of healthy donors as described [23], were incubated with different concentrations of Dendron 12 for 3 or 7 days. The apoptosis was monitored evaluating the percentage of dead cells by staining with 7-AAD (Beckman Coulter, Fullerton, California, USA). Flow cytometric analyses were performed using a CYTOMICS FC-500 flow cytometer interfaced with CXP21 software (Beckman Coulter).

\section{3-(4,5-Dimethylthiazol-2-yl)-2,5-diphenyltetrazolium bromide assay}

Toxicity of Dendron 12 was determined by a 3-(4,5-Dimethylthiazol-2-yl)-2,5diphenyltetrazolium bromide-based assay (Sigma-Aldrich). Viable explants reduce MTT to formazan crystals. Explants were cultured with increasing concentration of Dendron 12 diluted in medium culture for 3 and 7 days. The 50\% of medium culture (containing the different concentration of Dendron 12) was changed at day 3. After culturing, explants were washed and incubated in medium RPMI without phenol red and 10\% FBS and MTT. Formazan was dissolved by MTT solubilization solution and formazan absorbance was measured at $595 \mathrm{~nm}$.

\section{Differentiation and treatment of monocyte-derived dendritic cells}

CD14+ monocytes were separated from PBMCs using the CD14+ microbeads (Miltenyi Biotech, Bergisch Gladbach, Germany) following manufacturer's instruction. Monocytes were differentiated into iDCs by culturing them in presence of IL-4 $(20 \mathrm{ng} / \mathrm{ml})$ and granulocytemacrophage colony-stimulating factor $(20 \mathrm{ng} / \mathrm{ml})$ (R\&D Systems) for 6 days. DC-SIGN expression was checked by staining with anti human DC-SIGN-PE monoclonal antibody (clone 
AZND1, Beckman Coulter) and flow cytometric analysis. Monocyte-derived dendritic cells (MDDCs) were treated with Dendron 12. Supernatants were harvested and RNA extracted from cells after 3,24 and $72 \mathrm{~h}$. $\beta$ chemokine concentration in culture supernatants was evaluated using DuoSet kits (R\&D Systems).

\section{RNA extraction and real-time PCR}

RNA was extracted using the acid guanidium thiocyanate-phenol-chloroform method and purified from genomic DNA with RNase-free DNase (RQ1 DNase, Promega, Madison, Wisconsin, USA). RNA was reverse-transcribed using random examer primers and M-MLV reverse transcriptase (Clontech, Palo Alto, California, USA). cDNA quantification for macrophage inflammatory protein (MIP)-1 $\alpha$, MIP-1 $\beta$, RANTES (regulated upon activation, normal T-cell expressed, and secreted) and glycerAldehyde 3-phosphate dehydrogenase (GAPDH) was performed by real-time PCR (DNA Engine Opticon 2; MJ Research, Ramsey, Minnesota, USA). Reactions were performed using a SYBR Green PCR mix (Finnzymes, Espoo, Finland). Results were expressed as $\triangle \triangle \mathrm{Ct}$ and presented as ratios between the target gene and the GAPDH housekeeping mRNA.

\section{Surface plasmon resonance analysis}

Extracellular domain (ECD) of langerin (residue 68-328) and DC-SIGN (residue 66-404) was overexpressed and purified as described [28,29]. Surface plasmon resonance (SPR) experiments were performed on a Biacore 3000 using functionalized CM4 sensor chips and the corresponding reagents from Biacore. Two flow cells were activated as described [30]. Flow cell one was blocked with ethanolamine and used as a control surface. The second one was treated with BSA-Mana1-3[Mana1-6] Man (Man-BSA, Dextra) $(60 \mu \mathrm{g} / \mathrm{ml}$ ) in $10 \mathrm{mmol} / \mathrm{l}$ acetate buffer, $\mathrm{pH}$ 4. Remaining activated groups were blocked with ethanolamine. The final density immobilized on the surface of the second flow cell was 5200 RU. The Man-BSA used to functionalize CM4 chip harbors 12 glycosylation sites. The affinities for DC-SIGN ECD and langerin ECD of pseudo-mannotrioside and D-mannose were estimated by an inhibition assay, as described [22,31]. Each lectin was injected onto the Man-BSA surface, at $20 \mu \mathrm{mol} / \mathrm{l}$ alone or in presence of an increasing concentration of compounds. Injections were performed at 5 $\mu \mathrm{l} / \mathrm{min}$ using $25 \mathrm{mM}$ Tris- $\mathrm{HCl}, \mathrm{pH} 8,150 \mathrm{mM} \mathrm{NaCl}, 4 \mathrm{mmol} / \mathrm{l} \mathrm{CaCl}$, and $0.005 \%$ of $\mathrm{P} 20$ surfactant as running buffer. The surface was regenerated by $1^{\prime}$ injection of $50 \mathrm{mmol} / \mathrm{I}$ EDTA, $\mathrm{pH}$ 8. The IC50 values were determined as described $[22,31]$.

\section{Results}

Dendron 12 inhibits trans infection in presence of HIV-1 elevated viral load

We firstly examined whether the Dendron 12 was able to inhibit the trans infection in the presence of elevated viral load. B-THP1/DC-SIGN cells were used as model to mimic HIV transmission to CD4+ T cells, as previously described [6,23,32]. B-THP1/DC-SIGN cells were preincubated $30 \mathrm{~min}$ in the presence or in absence of Dendron 12 and then exposed to different inoculums of HIV-1 Bal. Then, B-THP1/DC-SIGN cells were washed and co-cultured with activated CD4+ T cells. At lower viral concentration inhibition of BaL transmission to CD4+ 
T cells was almost complete. Even at higher viral load (40 and 80 TCID50) the Dendron 12 was able to counteract the transmission of the virus to CD4+ T lymphocytes (more than $92 \%$ of inhibition) (Fig. 1a).

\section{Selectivity towards DC-SIGN}

Both langerin and DC-SIGN recognize Man9 on gp120, but have distinct specificities towards complex oligosaccharides [33-36]. Specificity of pseudo-mannotrioside ligand for langerin and DC-SIGN was tested by competition experiments using SPR, as previously described [37]. A CM4 sensor chip was functionalized with BSA-mannotriose and a fixed amount of the extracellular domain of DC-SIGN and langerin was injected over the surface in the presence or absence of pseudo-mannotrioside or mannose (control). From the inhibition curves (Fig. 1b), an IC50 of the two ligands towards each lectins was evaluated (Fig. 1c). A limited difference in favor of DC-SIGN was observed for mannose. On the contrary, pseudo-mannotrioside is 20 times more potent toward DC-SIGN than against langerin. Moreover, pseudo-mannotrioside is 14 times more potent than D-mannose towards DC-SIGN. Indeed, the use of pseudomannotrioside allows real improvement in affinity and in selectivity.

Inhibition of HIV-1 infection of human cervical tissue by Dendron 12

Endocervical tissue was obtained from premenopausal women, HIV, HBV and HCV seronegative, undergoing planned therapeutic hysterectomy. Explants were exposed to HIV-1 in a nonpolarized manner, analogous to condition of compromised epithelium in vivo. As the laboratory adapted R5 strain HIV-1 BaL is able to infect resting tissue [25], explants were not activated to mimic physiological conditions.

Explants were pretreated $30 \mathrm{~min}$ in absence or in presence of increasing concentration of Dendron 12 and then were exposed to BaL in the continued presence of compound. After washing to remove unbound Dendron 12 and virus, explants were maintained in culture up to 7 days. Data represent p24 levels and are presented as the mean of five independent experiments, using explants from separate donors. Dendron 12 inhibited cervical explants BaL infection in dose-dependent manner (Fig. 2). At the higher concentration tested, Dendron 12 reduced the infection by about $80 \%$, at 3 and 7 days post infection.

The ability of Dendron 12 to avert explant infection mediated by primary HIV-1 isolates was also verified. $\mathrm{R} 5$ tropic isolate $8 \mathrm{~g}$ was able to infect unstimulated tissue, but $\mathrm{X} 4$ tropic isolate DPMVF needed preactivation to induce infection (not shown). Explants unstimulated or preactivated 2 days with $\mathrm{IL}-2$ and PHA were pretreated with increasing concentration of Dendron 12 and infected, respectively, with $8 \mathrm{~g}$ or DPMVF, as described before. Infection inhibition was dose-dependent. At the concentration of $1 \mathrm{mmol} / \mathrm{l}$, infection by both isolates was reduced by more than $85 \%$. At $0.05 \mathrm{mmol} / \mathrm{I}$ Dendron 12 decreases by $56 \%$ (day 3 ) and by $40 \%$ (day 7) the infection mediated by $8 \mathrm{~g}$ (Fig. 3a and b), but the inhibitory effect against DPMVF was largely lost (Fig. $3 c$ and d).

Furthermore we evaluated the capability of Dendron 12 to block explant infection by Clade $C$ R5 tropic strain DU174. Unstimulated explants were pretreated with the compound, exposed 
to DU174 and cultured as described. Dendron 12 reduced DU174 infection in dose-dependent manner and by about $90 \%$ at $1 \mathrm{mM}$ (Fig. 3e and f).

Induction of $\beta 1$ chemokines production by Dendron 12

We wondered if, in addition to competitive inhibition of DC-SIGN, other mechanisms account for the antiviral effect of the compound 12. So we investigated if Dendron 12 stimulates the production of factors interfering with HIV infection. Due to difficulty of isolating sufficient amounts of primary mucosal dendritic cells, immature monocyte-derived dendritic cells (iMDDCs) that share with mucosal dendritic cells similar features and DC-SIGN expression, were used as a model [38]. iMDDCs were treated with Dendron 12 for 3,24 and $72 \mathrm{~h}$. Expression and production of $\beta$ chemokines MIP-1 $\alpha$, MIP-1 $\beta$, and RANTES following stimulation were analyzed by quantitative real-time PCR and ELISA. The treatment increased expression level of mRNA specific for MIP-1 $\alpha$, MIP-1 $\beta$ and RANTES (Fig. 4a-c) after $3 \mathrm{~h}$. Also MIP- $1 \alpha$, MIP- $1 \beta$ and RANTES production increased after 24 and $72 \mathrm{~h}$ of stimulation (Fig. $4 \mathrm{~d}$-f).

Evaluation of Dendron 12 toxicity

Cytotoxicity against PBMCs was evaluated by labeling with 7-amino-actinomycin D (7-AAD) that identifies nonviable cells after Dendron 12 treatment. Compound exposure for 3 and 7 days did not alter significantly the viability of PBMCs (Fig. 5a and b).

To assess toxicity of Dendron 12 towards cervical tissue, effect of nonpolarized exposure of the compound on explants viability was monitored (Fig. 5c). After 3 and 7 days treatment in absence or in presence of different concentration of Dendron 12, viability was evaluated by a MTT-based assay. Viability of the compound treated explants was compared to viability of untreated control. No significant difference between control and treated explants was observed up to a concentration of $1 \mathrm{mM}$ (the higher concentration tested in assessment of compound efficacy against HIV-1 infection).

\section{Discussion}

Three decades after HIV discovery HIV-AIDS pandemic continues and millions of people are infected every year. Thus, the development of effective, nontoxic and low-cost topical microbicides represents a valid alternative approach to prevent the sexual transmission of HIV [3]. However, so far almost all compounds failed to prevent HIV transmission in efficacy trials. A recent exception was a vaginal gel formulation of tenofovir that reduced HIV infection by $50 \%[39]$.

Tenofovir and other topical microbicides can prevent localized infection of target cells in genital mucosae. However, dendritic cells DC-SIGN+ internalize and transport HIV to secondary lymphoid organs, rendering the virus inaccessible to inhibitory effect of the microbicides. Therefore inhibition of DC-SIGN is essential to block HIV-1 uptake and dissemination from migratory dendritic cells. 
We have previously reported that the tetravalent Dendron 12 was able to block almost completely the HIV-1 trans infection of CD4+ T cells at micromolar range [23]. This compound exerts its activity by competitive inhibition of HIV-1 gp120 binding to DC-SIGN. In our initial experiments we demonstrated that Dendron 12 (at the same concentration previously assayed) even in presence of higher viral loads retains its ability to inhibit HIV-1 trans infection. This potent inhibitory activity is due both to elevate affinity for DC-SIGN of Dendron 12 pseudo-trisaccharide units and to high avidity of binding, guaranteed by the tetravalent presentation on the compound scaffold.

Different DC-SIGN inhibitors have been described so far. Dendrons displaying complex oligomannoses in high density inhibited binding of gp120 to DC-SIGN with IC50 in nanomolar range [40]. Nevertheless the complexity of the oligosaccharides used limits the possibility of a therapeutic application. Gold nanoparticles displaying mannosyl oligosaccharides are potent inhibitors of DC-SIGN-mediated HIV-1 trans infection of human PBMCs [41], but may have toxic effects because of gold accumulation.

Unlike DC-SIGN, the C-type lectin langerin, expressed on Langerhans cells, appears to play a protective role against HIV infection. Langerhans cells are located in the stratified mucosal epithelia of female and male genital tissue. Both DC-SIGN and langerin recognize high mannose glycans on gp120 through their carbohydrate recognition domain (CRD). Recent data demonstrate that langerin prevents HIV transmission by Langerhans cells, at least in the presence of low concentration of the virus, promoting rapid degradation and clearance of HIV$1[32,33]$. Using a biosensor with a SPR detection method we showed that the Dendron 12 is much more selective for DC-SIGN than for langerin. Considering the similarity between the CRD of DC-SIGN and langerin, the fact that the Dendron 12 does not interfere with langerin function is a remarkable advantage in view of developing new microbicides.

The efficacy and the safety of the Dendron 12 were evaluated in a human cervical explant model. Endocervix is more susceptible to HIV-1, being lined by a single layer of columnar epithelium, and contains dendritic cell DC-SIGN+ in the subepithelial region [4,5,24]. Explants were exposed to HIV-1 to mimic a condition of compromised epithelium in vivo; condition that highly increases the risk of HIV infection.

R5-tropic virus strains predominate during HIV-1 transmission in vivo and cause the majority of new infections [42]. The results obtained showed that Dendron 12 strongly reduced the infection of cervical explants by different HIV-1 R5 tropic strains, such as BaL, the primary isolate $8 \mathrm{~g}$ and the Clade C DU174. This may have a great impact, considering that Clade $\mathrm{C}$ is the most abundant subtype in all countries of Southern Africa and in some countries of eastern Africa and Asia, areas where the majority of HIV-1-infected people resides $[43,44]$.

Rare cases of HIV-1 infection by X4-tropic strains were observed in CCR5 $\triangle 32$ homozygotes and X4-tropic strain can infect immune activate human cervical tissue [25,42]. Dendron 12 was able to inhibit DPMVF primary X4-tropic strain infection at higher concentration assayed, but this effect was in part lost at lower concentrations of the compound. 
Data obtained suggest that Dendron 12 might have additional mechanisms of action apart from competitive inhibition of HIV-1 binding to DC-SIGN. Flow cytometric studies indicate that treatment with Dendron 12 reduces DC-SIGN expression on B-THP1/DC-SIGN+ cells [23]. This effect may be due to increased internalization of the receptor after binding of the compound to DC-SIGN.

Furthermore, Dendron 12 induced an increase of the production of $\beta$ chemokines, such as MIP- $1 \alpha$, MIP- $1 \beta$ and RANTES, by iMDDCs. These $\beta$ chemokines, natural ligands of CCR5, suppress HIV-1 R5 tropic strain replication, competing with the binding of the virus to CCR5 or inducing receptor internalization [45-47]. The Dendron 12, enhancing $\beta$ chemokines production, could interfere, at least partially, with the direct infection of CCR5+ CD4+ T lymphocytes and macrophages located in genital mucosae.

The Dendron 12 does not reduce the vitality of PBMCs and of explants. However, additional experiments, such as rabbit vaginal irritation assay [48], would be needed for a more accurate evaluation of potential toxic effects.

Vaginal epithelium has limited permeability to particles greater than $30 \mathrm{~nm}$ [2]. However, the diameter of Dendron 12 is certainly below that threshold, so the compound could enter and diffuse into intact mucosal tissue. Furthermore the Dendron 12 scaffold can be easily modified to improve absorption of the compound, without decreasing affinity to DC-SIGN.

The tetravalent Dendron 12 prevents HIV trans infection of CD4+ T lymphocytes at micromolar range, even in presence of elevated viral load, and displays high solubility in physiological media, a neglectable toxicity and a long-lasting effect. Moreover it inhibits in dose-dependent manner HIV-1 infection of human cervical explants. These features make the Dendron 12 a good candidate as a lead compound to develop new microbicide drugs.

However, the Dendron 12 inhibits $80-90 \%$ of cervical explants HIV-1 infection, but cannot block it completely. To overcome these limitations, the structure of this compound can be improved in both the scaffold and the active pseudo-saccharide ligand to develop new ligands of DC-SIGN more effective and easier to synthesize. Furthermore, Dendron 12 (or its derivatives) can be used in combination with other molecules directed against different HIV targets. In particular multivalent structures, presenting on the same scaffold multiple copies of DC-SIGN and HIV co-receptor inhibitors, simultaneously blocking different HIV targets, could protect against different routes of HIV transmission.

\section{Acknowledgements}

M.C., A. Bernardi, D.T. conceived the study; A. Berzi, M.C. wrote the paper, A. Berzi, M.B. performed the experiments and analyzed the data; Jo.Re., R.O. synthesized the pseudomannotrioside; Ja.Ro., M.S.N. synthesized the Dendron scaffold; F.F., I.S. performed SPR experiments and analyzed the data; E.C. expressed and purified DC-SIGN and langerin; I.C., P.A. obtained cervical explants.

Conflicts of interest 
Conflict of interest and source of funding: the authors declare no conflict of interest.

B-THP-1 and B-THP-1/DC-SIGN cells, HIV-1 BaL and DU174 were provided through the EU programme EVA centre for AIDS Reagents NIBSC, UK. Buffy coats from healthy donors were provided by the Transfusional Unit of Vimercate Hospital (Italy). This work was supported by: the FIRB program CHEM-PROFARMANET (RBPR05NWWC), Istituto Superiore di Sanità 'Programma Nazionale di Ricerca sull'AIDS', EMPRO and AVIP EC WP6 Projects, the nGIN EC WP7 Project, 2008 Ricerca Finalizzata and Ricerca Corrente (Italian Ministry of Health), the MICINN of Spain CTQ2008-01694/BQU European FP7 project: EU ITN CARMUSYS (PITN-GA2008-213592), Sidaction - ensemble contre le sida. A. Berzi was supported by a fellowship of Doctorate School of Molecular Medicine, University of Milan; I.S. by ITN Carmusys, E.C. by a fellowship from 'ministère de la recherché et de l'enseignement supérieur'; M.S.N. by FPU fellowship. 


\section{References}

1. UNAIDS Report on the global AIDS epidemic, 2010.

2. Cutler B, Justman J. Vaginal microbicides and the prevention of HIV infection. Lancet Infect Dis 2008; 8:685-697.

3. Reina JJ, Bernardi A, Clerici M, Rojo J. HIV microbicides: state-of-the-art and new perspectives on the development of entry inhibitors. Future Med Chem 2010; 2:1141-1159.

4. Geijtenbeek TBH, Torensma R, van Vliet SJ, van Duijnhoven GCF, Adema GJ, van Kooyk Y, et al. Identification of DC-SIGN, a novel dendritic cell-specific ICAM-3 receptor that supports primary immune responses. Cell 2000; 100:575-585.

5. Pope M, Haase AT. Transmission, cute HIV infection and the quest for strategies to prevent infection. Nat Med 2003; 9:847-852.

6. Wu L, Wu L, KevalRamani VL. Dendritic-cell interactions with HIV: infection and viral dissemination. Nat Rev Immunol 2006; 6:859-868.

7. Geijtenbeek TBH, Kwon DS, Torensma R, van Vliet SJ, van Duijnhoven GCF, Middel J, et al. DC-SIGN, a dendritic cell-specific HIV-1-binding protein that enhances trans-infection of T Cells. Cell 2000; 100:587-597.

8. Van Kooyk Y, Geijtenbeeck TBH. DC-SIGN: escape mechanism for pathogens. Nat Rev Immunol 2003; 3:697-709.

9. Kwon DS, Gregorio G, Bitton N, Hendrikson WA, Littman DR. DC-SIGN-mediated internalization of HIV is required for trans-enhancement of T cell infection. Immunity 2002; 16:135-144.

10. McDonald D, Wu L, Bohks SM, KewalRamani VN, Unutmaz D, Hope TJ. Recruitment of HIV and its receptors to dendritic cell-T cell junctions. Science 2003; 300:1295-1297.

11. Wiley RD, Gummuluru S. Immature dendritic cells derived exosomes can mediate HIV-1 trans infection. Proc Natl Acad Sci USA 2006; 103:738-743.

12. Lee B, Leslie G, Soilleux E, O'Doherty U, Baik S, Levroney E, et al. Cis expression of DC-SIGN allows for more efficient entry of human and simian immunodeficiency viruses via CD4 and a co-receptor. J Virol 2001; 75:12028-12038.

13. Turville SG, Santos JJ, Frank I, Cameron PU, Wilkinson J, Miranda-Saksena M, et al. Immunodeficiency virus uptake, turnover, and 2-phase transfer in human dendritic cells. Blood 2003; 103:2170-2179.

14. Gringhuis SI, den Dunnen J, Litjens M, van Het Hof B, van Kooyk Y, Geijtenbeek TBH. C-type lectin DC-SIGN modulates Toll-like receptor signaling via Raf-1 kinase-dependent acetylation of transcription factor NF-kB. Immunity 2007; 26:605-616. 
15. Hodges A, Sharrocks K, Edelmann M, Baban D, Moris A, Schwartz O, et al. Activation of the lectin DC-SIGN induces an immature dendritic cell phenotype triggering Rho-GTPase activity required for HIV-1 replication. Nat Immunol 2007; 8:569-577.

16. Gringhuis $\mathrm{SI}$, van der Vlist $M$, van den Berg LM, den Dunnen J, Litjens $M$, Geijtenbeek TBH. HIV-1 exploits innate signaling by TLR8 and DC-SIGN for productive infection of dendritic cells. Nat Immunol 2010; 11:419-426.

17. Gringhuis SI, den Dunnen J, Litjens M, van der Vlist M, Geijtenbeek TBH. Carbohydratespecific signaling through the DC-SIGN signalosome tailors immunity to Mycobacterium tuberculosis, HIV-1 and Helicobacter pylori. Nat Immunol 2010; 10:1081-1089.

18. Feinberg H, Mitchell DA, Drickamer K, Weis WI. Structural basis for selective recognition of oligosaccharides by DC-SIGN and DC-SIGNR. Science 2001; 294:2163-2166.

19. Adams EW, Ratner DM, Bokesch HR, McMahon JB, O'Keefe BR, Seeberger PH. Oligosaccharide and glycoprotein microarrays as tools in HIV glycobiology; glycan-dependent gp120/protein interactions. Chem Biol 2004; 11:875-881.

20. Reina JJ, Sattin S, Invernizzi D, Mari S, Martínez-Prats L, Tabarani G, et al. 1,2 Mannobioside mimic: synthesis, DC-SIGN interaction by NMR and docking, and antiviral activity. Chem MedChem 2007; 2:1030-1036.

21. Rojo J, Delgado R. Glycodendritic structures: promising new antiviral drugs. J Antimicrob Chemother 2004; 54:579-581.

22. Luczkowiak J, Sattin S, Sutkevičiūte I, Reina JJ, Sánchez-Navarro $M$, Thépaut $M$, et al. Pseudosaccharide functionalized dendrimers as potent inhibitors of DC-SIGN dependent Ebola pseudotyped viral infection. Bioconj Chem 2011; 22:1354-1365.

23. Sattin S, Daghetti A, Thépaut M, Berzi A, Sanchez-Navarro M, Rojo J, et al. Inhibition of DCSIGN-mediated infecion by a linear trimannoside mimic in a tetravalent presentation. ACS Chem Biol 2010; 5:301-312.

24. Anderson DJ, Pudney J, Shust DJ. Caveat associated with the use of human cervical tissue for HIV and microbicide research. AIDS 2010; 24:1-4.

25. Greenhead P, Hayes P, Watts P, Laing K, Griffin G, Shattock R. Parameters of Human Immunodeficiency Virus Infection of human cervical tissue and inhibition by vaginal virucides. J Virol 2000; 74:5577-5586.

26. Wallace GS, Cheng-Mayer C, Schito ML, Fletcher P, Miller Jenkins LM, Hayashi $R$, et al. Human immunodeficiency virus type 1 nucleocapsid inhibitors impede trans infection in cellular and explant models and protect nonhuman primates from infection. J Virol 2009; 83:9175-9182.

27. Cummins JE, Guarner J, Flowers L, Guenthner PC, Bartlett J, Morken T, et al. Preclinical testing of candidate topical microbicides for antihuman immunodeficiency virus type 1 activity 
and tissue toxicity in a human cervical explant culture. Antimicrob Agents Chemother 2007; 51:1770-1779.

28. Thépaut $M$, Valladeau J, Nurisso A, Kahn R, Arnou B, Vivès $C$, et al. Structural studies of Langerin and Birbeck granule: a macromolecular organization model. Biochemistry 2009; 48:2684-2698.

29. Tabarani G, Thépaut M, Stroebel D, Ebel C, Vivès $C$, Vachette $P$, et al. DC-SIGN neck domain is a $\mathrm{pH}$-sensor controlling oligomerization: SAXS and hydrodynamic studies of extracellular domain. J Biol Chem 2009; 284:21229-21240.

30. Halary $F$, Amara A, Lortat-Jacob $H$, Messerle $M$, Delaunay $T$, Houlès $C$, et al. Human cytomegalovirus binding to DC-SIGN is required for dendritic cell infection and target cell transinfection. Immunity 2002; 17:653-664.

31. Andreini M, Doknic D, Sutkeviciute I, Reina JJ, Duan J, Chabrol E, et al. Second generation of fucose-based DC-SIGN ligands: affinity improvement and specificity versus Langerin. Org Biomol Chem 2011; 9:5778-5786.

32. Wu L, Martin TD, Carrington M, KewalRamani VN. Raji B cells, misidentified as THP-1 cells, stimulate DC-SIGN- mediated HIV transmission. Virology 2004; 318:17-23.

33. de Witte L, Nabatov A, Pion M, Fluitsma D, Marein AW, de Jong $P$, et al. Langerin is natural barrier to HIV-1 transmission by langerhans cells. Nat Med 2007; 13:367-371.

34. de Witte L, Nabatov A, Geijtenbeek TBH. Distinct roles for DC-SING-dentritic cells and langherans cells in HIV-1 transmission. Trends Mol Med 2007; 14:12-19.

35. Guo Y, Feinberg H, Conroy E, Mitchell DA, Alvarez R, Blixt O, et al. Structural basis for distinct ligand-binding and targeting properties of the receptors DC-SIGN and DC-SIGNR. Nat Struct Mol Biol 2004; 11:591-598.

36. Galustian C, Park CG, Chai W, Kiso M, Bruening SA, Kang YS, et al. High and low affinity carbohydrate ligands revealed for murine SIGN-R1 by carbohydrate array and cell binding approaches, and differing specificities for SIGN-R3 and Langerin. Int Immunol 2004; 16:853866.

37. Timpano G, Tabarani G, Anderluh M, Invernizzi D, Vasile F, Potenza D, et al. Synthesis of novel DC-SIGN ligands with an alpha-fucosylamide anchor. ChemBioChem 2008; 9:1921-1930.

38. Sallusto F, Lanzavecchia A. Efficient presentation of soluble antigen by cultured human dendritic cells is maintained by granulocyte/macrophage colony-stimulating factor plus interleukin 4 and downregulated by tumor necrosis factor alpha. J Exp Med 1994; 179:11091118.

39. Abdool Karim Q, Abdool Karim SS, Frohlich JA, Grobler AC, Baxter C, Mansoor LE, et al. Effectiveness and safety of tenofovir gel, an antiretroviral microbicide, for the prevention of HIV infection in women. Science 2010; 329:1168-1174. 
40. Wang SK, Liang PH, Astronomo RD, Hsu TL, Hsieh SL, Burton DR, et al. Targeting the carbohydrates on HIV-1: interaction of oligomannose dendrons with human monoclonal antibody 2 G12 and DC-SIGN. Proc Natl Acad Sci USA 2008; 105:3690-3695.

41. Martinez-Avila O, Bedoya LM, Marradi M, Clavel C, Alcami J, Penades S. Multivalent manno-glyconanoparticles inhibit DC-SIGN-mediated HIV-1 trans-infection of human T cells. Chem Biochem 2009; 10:1806-1809.

42. Nazari R, Joshi S. CCR5 as target for HIV-1 gene therapy. Curr Gene Ther 2008; 8:1-9.

43. McCutchan FE. Understanding the genetic diversity of HIV-1. AIDS 2000; 14 (Suppl 3):S31S44.

44. Spira S, Wainberg MA, Loemba H, Turner D, Brenner BG. Impact of clade diversity on HIV-1 virulence, antiretroviral drug sensitivity and drug resistance. J Antimicrob Chemother 2003; 51:229-240.

45. Samson M, Libert F, Doranz BJ, Rucker J, Liesnard C, Farber CM, et al. Resistance to HIV-1 infection in Caucasian individuals bearing mutant alleles of the CCR-5 chemokine receptor gene. Nature 1996; 26:722-725.

46. Wu L, LaRosa G, Kassam N, Gordon CJ, Heath H, Ruffing N, et al. Interaction of chemokine receptor CCR5 with its ligands: multiple domains for HIV-1 gp120 binding and a single domain for chemokine binding. J Exp Med 1997; 186:1373-1381.

47. Cocchi F, DeVico AL, Garzino-Demo A, Arya SK, Gallo RC, Lusso P. Identification of RANTES, MIP-1(, and MIP-1( as the major HIV-1-suppressive factors produced by CD8 T cells. Science 1995; 270:1811-1815.

48. Eckstein P, Jackson MC, Millman N, Sobrero AJ. Comparison of vaginal tolerance tests of spermicidal preparations in rabbits and monkeys. J Reprod Fertil 1969; 20:85-93.

\section{Keywords:}

cervical explants; DC-SIGN; glycomimetic drugs; HIV; topical microbicides 


\section{Figure captions}

Figure 1. Evaluation of Dendron 12 ability to inhibit HIV-1 Bal trans infection in presence of increasing amounts of the virus (a) and selectivity to DC-SIGN (b,c).(a) After pretreatment with Dendron $12(250 \mu \mathrm{m})$ or medium culture, B-THP-1/DC-SIGN cells were pulsed with BaL for $3 \mathrm{~h}$. After washing cells were co-cultured 3 days with $C D 4+T$ lymphocytes from healthy donors. Levels of infection were quantified by measuring p24 in the supernatants of co-cultures by ELISA. Data were obtained from three different healthy donors. Each donor was tested in duplicate. Values are mean \pm SD. (b, c) SPR experiment results of DC-SIGN ECD and langerin ECD binding to Man-BSA/dextran surface inhibition by pseudo-mannotrioside (psTri) and Dmannose. (b) Inhibition curves, and (c) lectin selectivity histograms.

Figure 2. Inhibition of endocervical tissue infection mediated by HIV-1 Bal.After a $30 \mathrm{~min}$ pretreatment with the Dendron 12 or medium culture, endocervical explants were incubated $3 \mathrm{~h}$ with $\mathrm{BaL}$ in the continued presence of indicated concentrations of Dendron 12 . Then explants were washed and cultured for 7 days. Infection was monitored by ELISA measurement of p24 in explant culture supernatants at day 3 (a) and 7 (b) post infection. Values represent the mean $\pm S D$ of five independent experiments. ${ }^{*} \mathrm{P}<0.05$ (Student's t-test), $* * \mathrm{P}<0.01,{ }^{* * *} \mathrm{P}<0.001$.

Figure 3. Inhibition of endocervical infection induced by HIV-1 primary isolates $(8 \mathrm{~g}$ and DPMVF) and HIV-1clade C DU174.(a, b, e, f) Unstimulated explants were pretreated 30' with Dendron 12 or medium culture and challenged with $8 \mathrm{~g}$ or DU174. (c, d) Explants immunostimulated were pretreated 30' with Dendron 12 or medium culture and infected with DPMVF. After washing explants were put in culture. Infection was monitored by ELISA measurement of p24 in explant culture supernatants at day 3 and 7 post infection. Values represent the mean $\pm S D$ of three independent experiments. ${ }^{*} P<0.05$ (Student's $t$-test).

Figure 4. $\beta$ chemokines induction upon Dendron $12(250 \mu \mathrm{mol} / \mathrm{l})$ stimulation of human iMDDCs from healthy donors. $(\mathrm{a}-\mathrm{c}) \beta$ chemokines expression following $3 \mathrm{~h}$ Dendron $12(250 \mu \mathrm{mol} / \mathrm{l})$ treatment. mRNA levels of MIP-1 $\alpha$ (a), MIP-1 $\beta$ (b) and RANTES (c) were assessed by quantitative real-time PCR. Expression is normalized to GAPDH and shown as fold changes expression from the unstimulated sample, set as 1 . (d-f) $\beta$ chemokines protein production

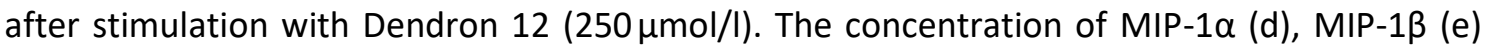
and RANTES (f) in the culture supernatants was assayed by ELISA at 24 and $72 \mathrm{~h}$. (a-f) Values represent the mean \pm SD.

Figure 5. Toxicity of compound 12.(a, b) Percentage of 7-AAD-positive (nonviable) PBMCs after 3 or 7 days of incubation with different concentrations of Dendron $12(0.25,0.5$ and $0.1 \mathrm{mmol} / \mathrm{l}$ ), or in the absence of the inhibitor (medium). Percentage of 7-AAD was determined by flow cytometry. Experiments were performed on PBMCs from three healthy donors. Values are mean \pm SD. Effect of Dendron 12 on cervical explants viability (c). Endocervical explants, derived from the same donors, were exposed in nonpolarized manner to Dendron 12 or culture medium (control) for 3 and 7 days. Effect of Dendron 12 on tissue viability was determined by the MTT assay. Explants were weighted to normalize optical density of formazan yielded. Viability was expressed as percentage. Experiments were performed on explants from three donors. Data are reported as the average percentage viability ( $\pm S D$ ). 
Figure 1

[a]

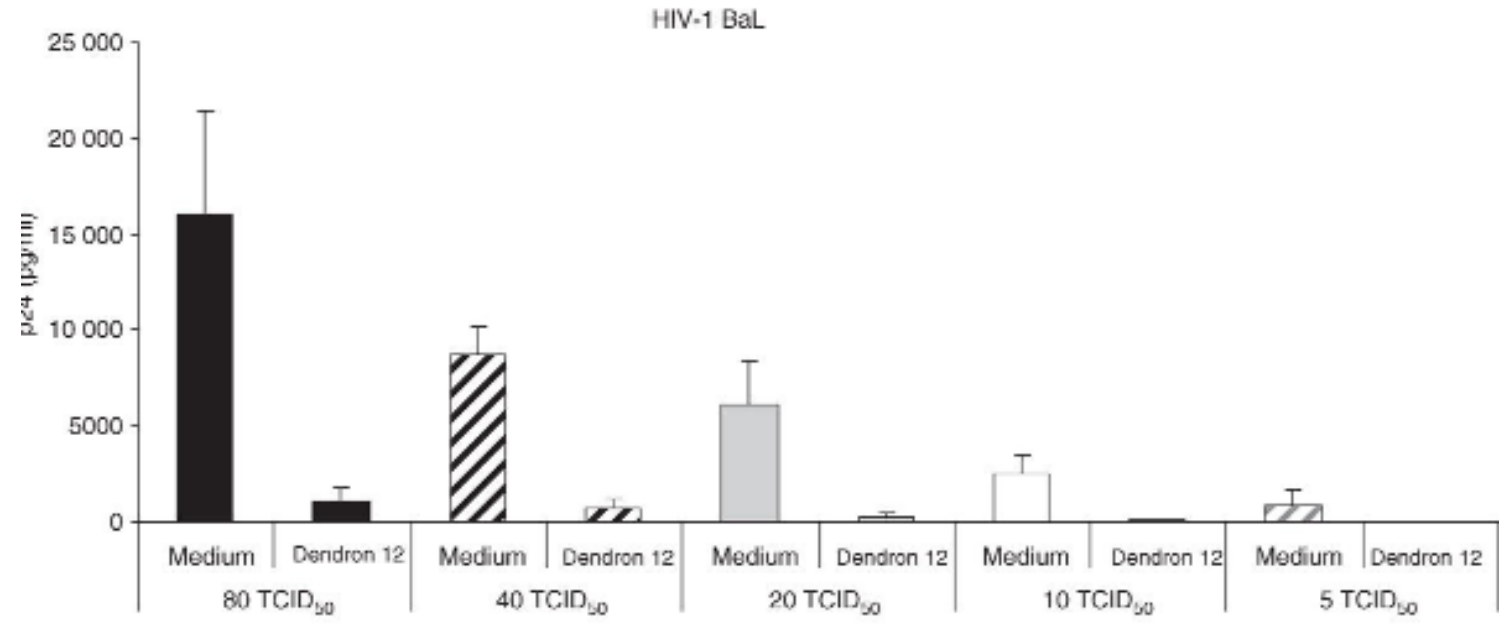

(b)

(c)
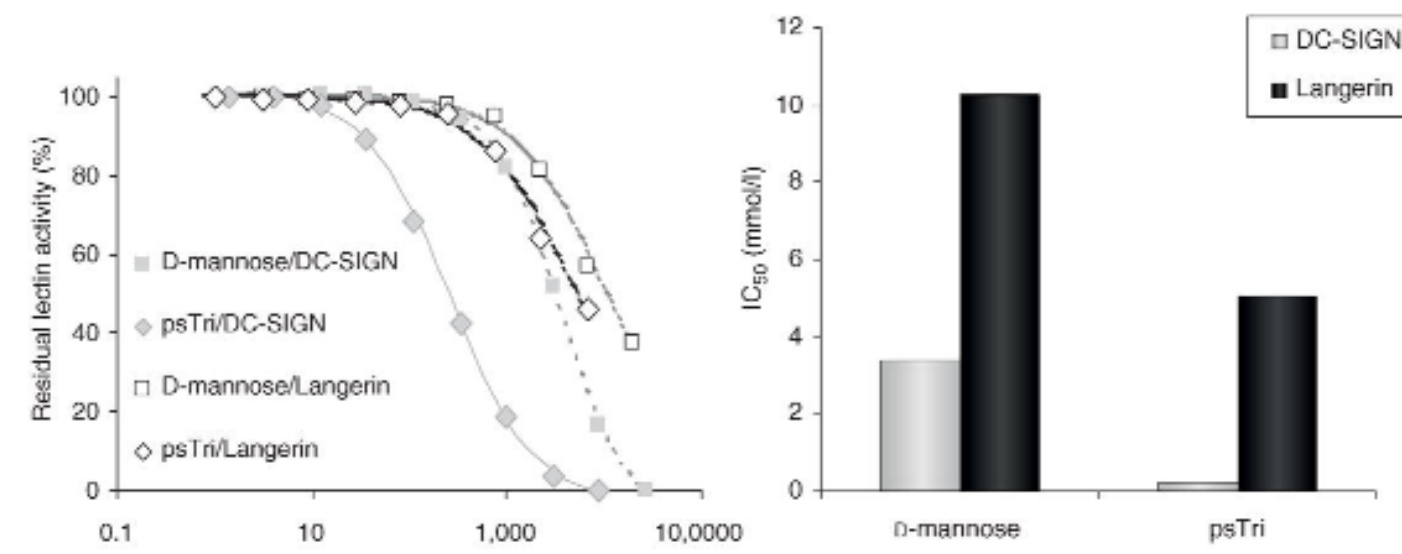

Compound concentration (umolil) 
Figure 2

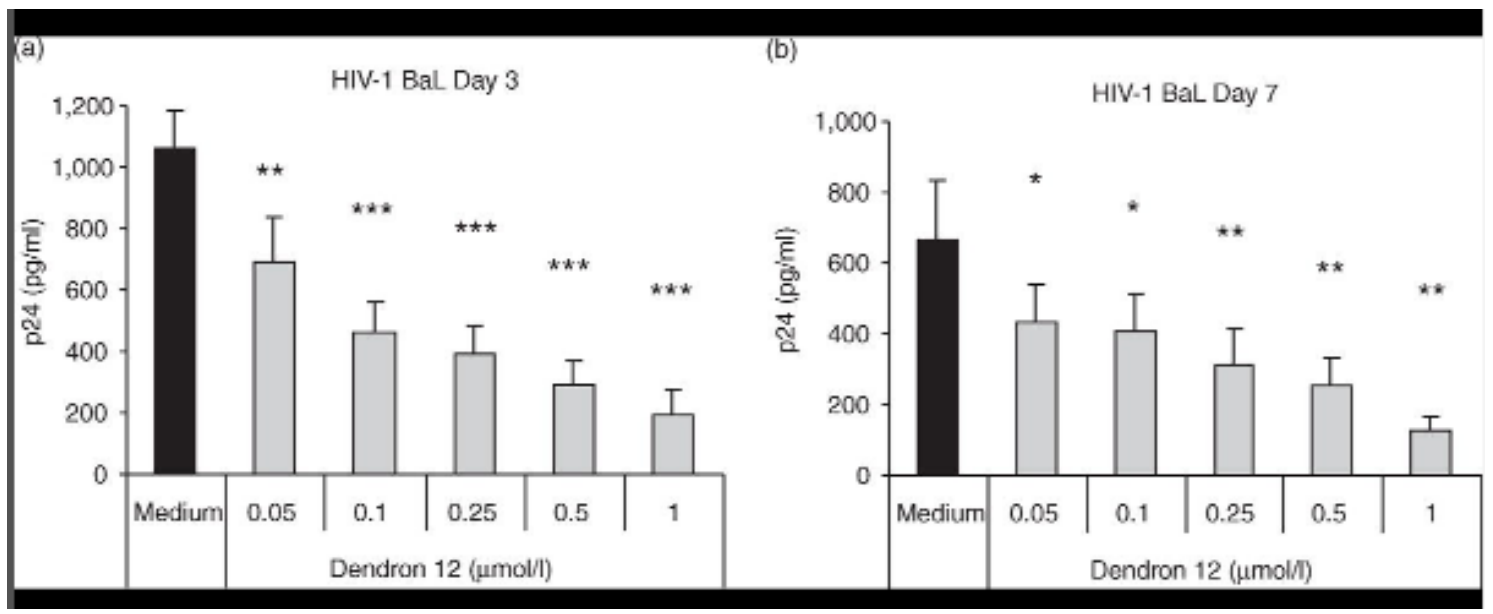


Figure 3

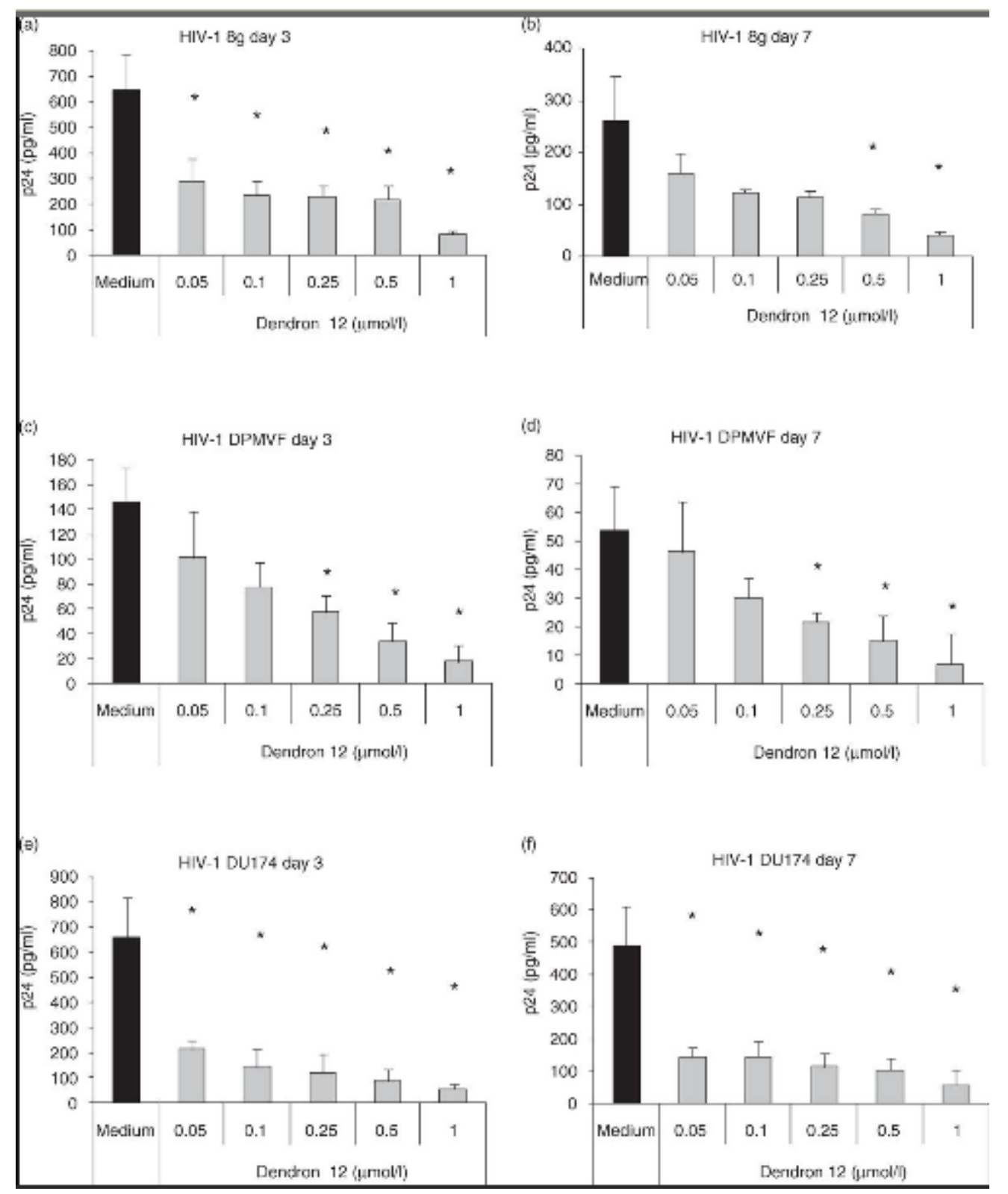


Figure 4

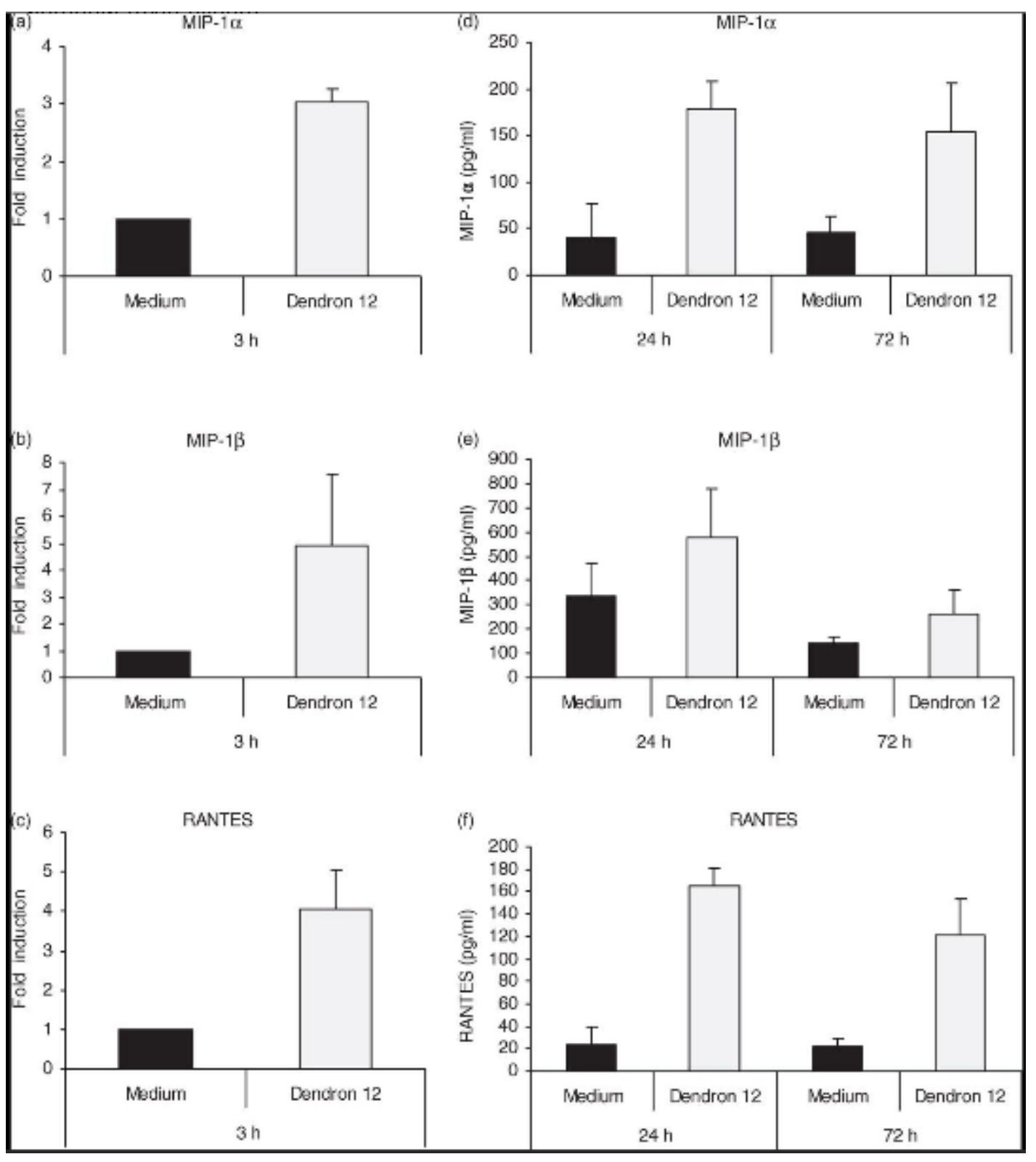


Figure 5

(a)

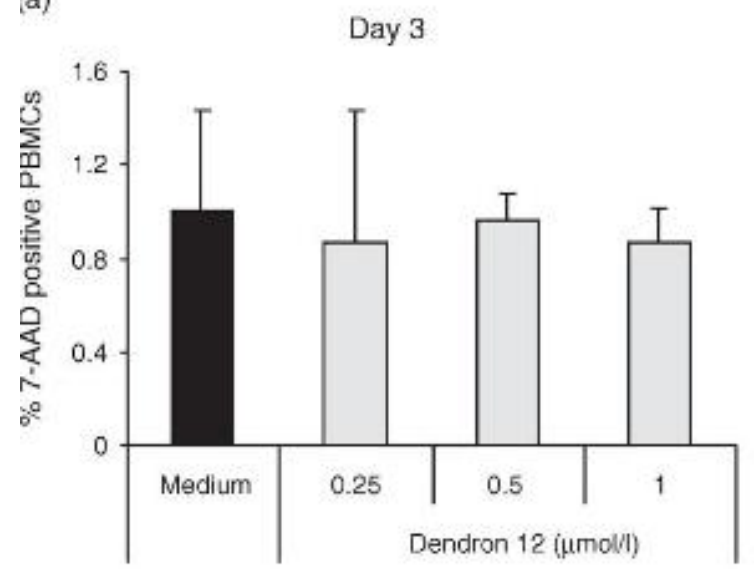

(b)

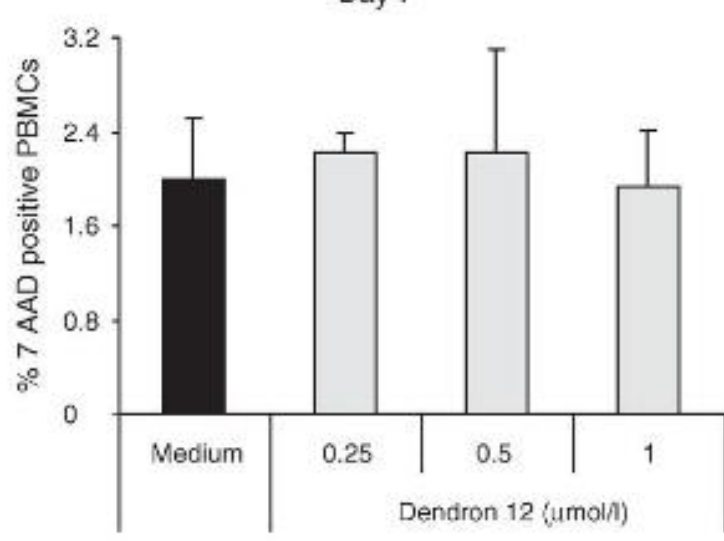

(c)

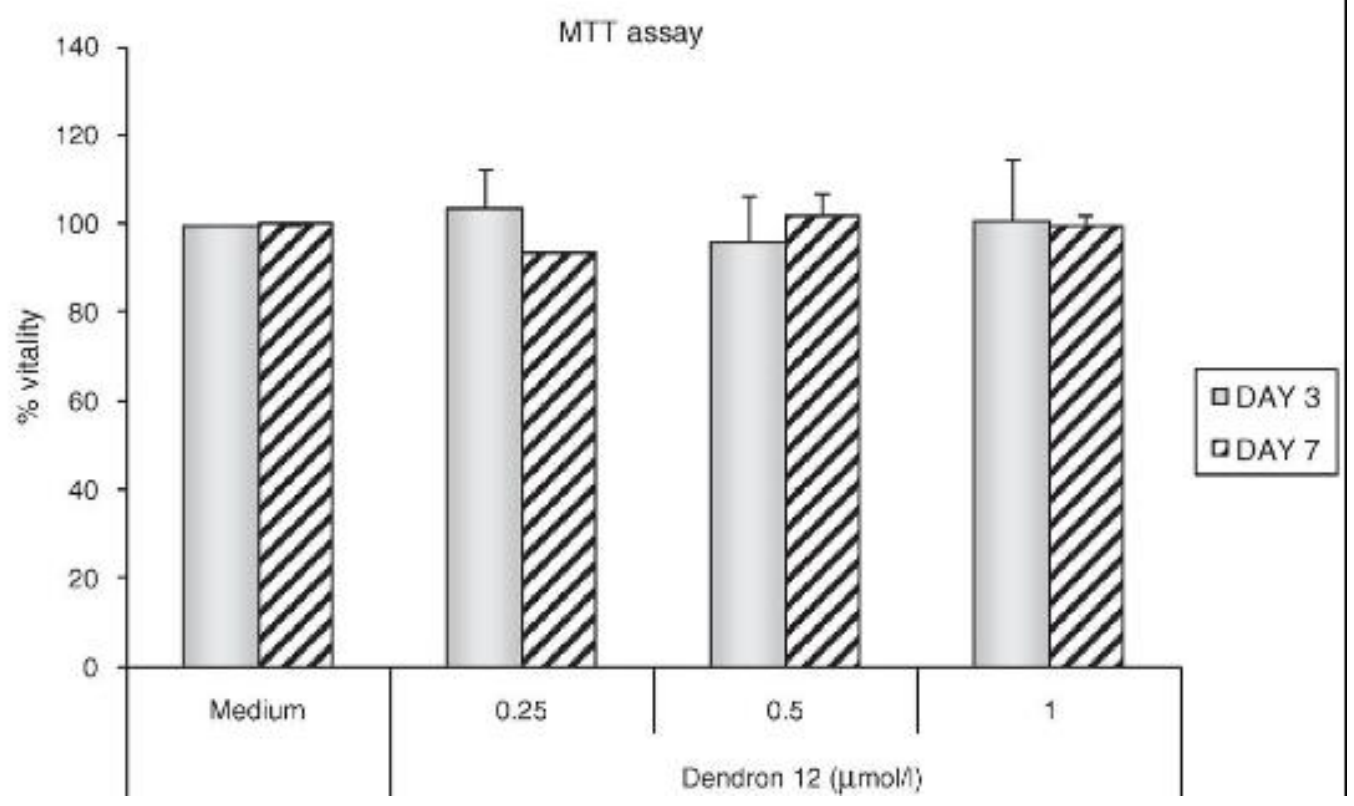

